\title{
MODULE CATEGORIES WITHOUT SHORT CYCLES ARE OF FINITE TYPE
}

\author{
DIETER HAPPEL AND SHIPING LIU
}

(Communicated by Maurice Auslander)

\begin{abstract}
Let $A$ be an artin algebra. An indecomposable finitely generated $A$ module $X$ is said to be on a short cycle if there exists an indecomposable finitely generated $A$-module $Y$ and two nonzero noninvertible maps $f: X \rightarrow Y$ and $g: Y \rightarrow X$. If there are no short cycles we show that there exist only finitely many indecomposable $A$-modules up to isomorphism.
\end{abstract}

Throughout this paper $A$ will denote an artin algebra over a commutative artin ring $R, \bmod A$ the category of finitely generated left $A$-modules, and $\mathfrak{R}$ the Jacobson radical of $\bmod A$. The artin algebra $A$ is said to be of finite representation type if there exist only finitely many indecomposable modules in $\bmod A$ up to isomorphism. We do not distinguish between an indecomposable module $X$ in $\bmod A$ and its isomorphism class. A path in $\bmod A$ is a sequence $\left(X_{0}, X_{1}, \ldots, X_{s}\right)$ of indecomposable modules in $\bmod A$ such that $\mathfrak{R}\left(X_{i-1}, X_{i}\right) \neq 0$ for all $1 \leq i \leq s$. If $s \geq 1$ and $X_{0}=X_{s}$, then the path $\left(X_{0}, X_{1}, \ldots, X_{s}\right)$ is called a cycle in $\bmod A$. If $s=2$ and $X_{0}=X_{2}$, then the path $\left(X_{0}, X_{1}, X_{2}\right)$ is called a short cycle in $\bmod A$. An indecomposable module $X$ in $\bmod A$ is said to be directing if it does not occur in any cycle in $\bmod A$.

The aim of this note is to show that if $A$ is an artin algebra such that $\bmod A$ contains no short cycle, then $A$ is of finite representation type.

This generalizes the following result by Ringel $\left[8,\left(2.4 .9^{\prime}\right)\right]$. A finite-dimensional algebra $A$ over an algebraically closed field is of finite representation type if every indecomposable module in $\bmod A$ is directing.

We point out that the proof of our result is obtained from a combination of Ringel's methods with methods and results of [3] and [7].

Note that the class of artin algebras whose module categories contain no short cycle is substantially larger than that of artin algebras whose module categories contain no cycle (see [3]).

We keep the notation introduced as before. The composition of two maps $f: X \rightarrow Y$ and $g: Y \rightarrow Z$ is denoted by $f g$; so we write maps in $\bmod A$ on the right. We denote by $\Gamma_{A}$ the Auslander-Reiten quiver of $A$ and by $\tau$ the Auslander-Reiten translation DTr. Recall from [3] that an indecomposable

Received by the editors March 30, 1992 and, in revised form, May 26, 1992.

1991 Mathematics Subject Classification. Primary 16G10, 16G60.

(C) 1994 American Mathematical Society $0002-9939 / 94 \$ 1.00+\$ .25$ per page 
module $X$ in $\bmod A$ is said to be in the middle of a short chain if there exists a chain $Y \rightarrow X \rightarrow \tau Y$ of nonzero maps in $\bmod A$ where $Y$ is a nonprojective indecomposable module. The following observation is useful.

Lemma 1. Let $A$ be an artin algebra, and let $X$ be an indecomposable module in $\bmod A$. Then the following are equivalent:

(a) $X$ is on a short cycle in $\bmod A$.

(b) $X$ is in the middle of a short chain in $\bmod A$.

Proof. It is shown in [7, (1.6)] that (b) implies (a). For the converse, assume that $\bmod A$ contains a short cycle $(X, Y, X)$, say, with nonzero nonisomorphisms $f: X \rightarrow Y$ and $g: Y \rightarrow X$. If $f g=0$, then there exists by [5, $\S 1]$ a nonprojective indecomposable module $W$ with $\operatorname{Hom}(W, X) \neq 0$ and $\operatorname{Hom}(X, \tau W) \neq 0$. So $X$ is in the middle of a short chain. If $f g \neq 0$, then let $t>1$ be the minimal positive integer such that $(f g)^{t}=0$. Let $f^{\prime}=f g$ and $g^{\prime}=(f g)^{t-1}$. Then $f^{\prime} g^{\prime}=0$. Applying the result in [5] again, we get that $X$ is in the middle of a short chain.

In the next lemma we will derive some homological properties of module categories without short cycles.

Lemma 2. Let $A$ be an artin algebra such that $\bmod A$ contains no short cycle. Let $X$ be an indecomposable module in $\bmod A$. Then $\operatorname{End}(X)$ is a division ring, and $\operatorname{Ext}_{A}^{1}(X, X)=\operatorname{Ext}_{A}^{2}(X, X)=0$.

Proof. It is easy to see that $\operatorname{End}(X)$ is a division ring, since $X$ is not on a short cycle. Moreover, $\operatorname{Hom}(X, \tau X)=0$, since $X$ is not in the middle of a short chain by Lemma 1. Being an epimorphic image of $\operatorname{Hom}(X, \tau X)$, $\operatorname{Ext}_{A}^{1}(X, X)=0$. For the last assertion, let $0 \rightarrow X \rightarrow I \rightarrow X^{\prime} \rightarrow 0$ be an exact sequence in $\bmod A$, with $I$ the injective envelope of $X$. Then $\operatorname{Ext}_{A}^{2}(X, X) \cong \operatorname{Ext}_{A}^{1}\left(X, X^{\prime}\right)$. Let $I^{\prime}$ be an indecomposable summand of $I$. Then $\operatorname{Hom}\left(X, I^{\prime}\right) \neq 0$. Since $I^{\prime}$ is not in the middle of a short chain, we see that $\operatorname{Hom}\left(I^{\prime}, \tau X\right)=0$; hence, $\operatorname{Hom}\left(X^{\prime}, \tau X\right)=0$. Therefore, $\operatorname{Ext}_{A}^{1}\left(X, X^{\prime}\right)=$ 0 , since it is an epimorphic image of $\operatorname{Hom}\left(X^{\prime}, \tau X\right)$.

Let ${ }_{s} \Gamma_{A}$ be the full subquiver of $\Gamma_{A}$, obtained by deleting all modules whose $\tau$-orbits contain either projective modules of injective modules and all arrows attached to these modules. Then the connected components of the quiver ${ }_{s} \Gamma_{A}$ are called stable components of $\Gamma_{A}$. A stable component $\Gamma$ of $\Gamma_{A}$ is said to be $\tau$-periodic if $\Gamma$ contains only $\tau$-periodic modules.

Lemma 3. Let $A$ be an artin algebra. If $\Gamma_{A}$ has infinitely many $\tau$-orbits, then $\Gamma_{A}$ has at least one infinite stable component.

Proof. Assume that $\Gamma_{A}$ contains infinitely many $\tau$-orbits. Let $\Gamma$ be a finite connected component of ${ }_{s} \Gamma_{A}$. Then $\Gamma$ is not a connected component of $\Gamma_{A}$, since $A$ is of infinite representation type [1]. By definition of ${ }_{s} \Gamma_{A}$ we infer that $\Gamma$ intersects with the $\tau$-orbit of a module which is an immediate predecessor of some projective or injective module. Since $\Gamma_{A}$ is locally finite and contains only finitely many projective and finitely many injective modules, we conclude that the union of all finite connected components of ${ }_{s} \Gamma_{A}$ contains only finitely many $\tau$-orbits of $\Gamma_{A}$. Hence $\Gamma_{A}$ has at least one infinite stable component, since $\Gamma_{A}$ contains infinitely many $\tau$-orbits. 
Let $f: X \rightarrow Y$ be an irreducible map in $\bmod A$. Recall from [6] that the left degree $d_{l}(f)$ of $f$ is infinite if, for each integer $n \geq 0$ and each map $g \in \mathfrak{R}^{n} \backslash \mathfrak{R}^{n+1}$, we have that $g f \notin \mathfrak{R}^{n+2}$; otherwise, $d_{l}(f)$ is the least integer $m$ such that there is a map $g$ in $\mathfrak{R}^{m} \backslash \mathfrak{R}^{m+1}$ such that $g f \in \mathfrak{R}^{m+2}$.

Let

$$
X_{0} \stackrel{f_{1}}{\longrightarrow} X_{1} \rightarrow \cdots \rightarrow X_{n-1} \stackrel{f_{n}}{\longrightarrow} X_{n}
$$

be a chain of irreducible maps in mod $A$. It follows easily from the definition that if $d_{l}\left(f_{i}\right) \geq n$ for all $1 \leq i \leq n$, then the composition $f_{1} \cdots f_{n}$ is in $\mathfrak{R}^{n} \backslash \mathfrak{R}^{n+1}$.

Lemma 4. Let $A$ be an artin algebra. Assume that each $\tau$-orbit of $\Gamma_{A}$ contains only finitely many modules. Then either $A$ is of finite representation type, or mod $A$ contains short cycles.

Proof. Assume that $A$ is of infinite representation type. Then it follows from our assumption that $\Gamma_{A}$ has infinitely many $\tau$-orbits. By Lemma 3 there exists an infinite stable component $\Gamma$ of $\Gamma_{A}$. It is clear that $\Gamma$ contains only $\tau$ periodic modules. Then it follows from [4] that $\Gamma$ is a stable tube, say, of rank $r$. Let $X$ be a module in $\Gamma$ with quasi-length $2 r$, and let $Y \rightarrow X$ be the arrow pointing to the mouth. Choose irreducible maps $f_{i}: \tau^{i} Y \rightarrow \tau^{i} X$ and $g_{i}: \tau^{i+1} X \rightarrow \tau^{i} Y$ for $0 \leq i \leq r-1$. Since $X$ has quasi-length $2 r$, there exists a sectional path

$$
Z_{1} \rightarrow Z_{2} \rightarrow \cdots \rightarrow Z_{2 r-1} \rightarrow Z_{2 r}=X
$$

in $\Gamma$ such that $Z_{2 r-1} \oplus Y$ is a summand of the middle term of the almost split sequence ending with $X$. Hence by $[6,(1.6)]$ the left degree of $f_{0}$ is greater than or equal to $2 r$. The same argument shows that all irreducible maps $f_{i}$ and $g_{i}$ with $0 \leq i \leq r-1$ have left degree greater than or equal to $2 r$. Hence the composition $g_{r-1} f_{r-1} \cdots g_{0} f_{0}$ is in $\mathfrak{R}^{2 r} \backslash \mathfrak{R}^{2 r+1}$. Thus we get a short cycle in $\bmod A$.

Recall that a module $X$ in $\bmod A$ is sincere if every simple module in $\bmod A$ occurs as a composition factor of $X$.

Theorem. Let $A$ be an artin algebra such that $\bmod A$ contains no short cycle. Then $A$ is of finite representation type.

Proof. As in $\left[8,\left(2.4 .9^{\prime}\right)\right]$ we may assume that there exists a sincere indecomposable module $X$ in $\bmod A$. Then it follows from [7, (3.5)] that the global dimension of $A$ is at most two. Let $q_{A}$ be the homological quadratic form on the Grothendieck group $K_{0}(A)$ of $A$. For a module $Z$ in $\bmod A$ we denote by [Z] the corresponding element in $K_{0}(A)$, and for an $R$-module $M$ we denote by $l_{R}(M)$ the length of $M$ over $R$. Then

$$
q_{A}([Z])=l_{R}(\operatorname{Hom}(Z, Z))-l_{R}\left(\operatorname{Ext}_{A}^{1}(Z, Z)\right)+l_{R}\left(\operatorname{Ext}_{A}^{2}(Z, Z)\right) .
$$

Note that $q_{A}$ is a quadratic form with integral coefficients. Assume that $K_{0}(A)$ is of rank $n$. We identify $K_{0}(A)$ with $\mathbf{Z}^{n}$.

An element $0 \neq x=\left(x_{1}, \ldots, x_{n}\right) \in \mathbf{Z}^{n}$ with $x_{i} \geq 0$ is called positive. A quadratic form $q$ on $\mathbf{Z}^{n}$ is called weakly positive if $q(x)>0$ for all positive $x$. 
We claim that $q_{A}$ is weakly positive. Indeed the usual argument applies. Let $0 \neq x=\left(x_{1}, \ldots, x_{n}\right) \in Z^{n}$ with $x_{i} \geq 0$. Choose a module $Z$ in $\bmod A$ such that $[Z]=x$ and $l_{R}\left(\operatorname{End}_{A}(Z)\right)$ is minimal among all those modules $Y$ in $\bmod A$ with $[Y]=x$. Assume that $Z=\bigoplus_{1}^{r} Z_{i}$ with the $Z_{i}$ indecomposable. Then by Lemma 2 we have that $\operatorname{Ext}_{A}^{1}\left(Z_{i}, Z_{i}\right)=0$ for all $1 \leq i \leq r$, and by [8, Lemma 1. (2.3)] we have that $\operatorname{Ext}_{A}^{1}\left(Z_{i}, Z_{j}\right)=0$ for all $i \neq j$. Thus

$$
q_{A}(x)=q_{A}([Z])=l_{r}(\operatorname{End}(Z))+l_{R}\left(\operatorname{Ext}_{A}^{2}(Z, Z)\right)>0,
$$

since $Z \neq 0$.

Let $t \in \mathbf{Z}$. By applying the argument used in [8, (1.0.2)] to prove a result of Drozd's, one can easily see that there exist only finitely many positive $x$ in $\mathbf{Z}^{n}$ with $q_{A}(x)=t$. We are now going to show that the number of modules in each $\tau$-orbit of $\Gamma_{A}$ is finite. Let $U$ and $V$ be modules in the same $\tau$-orbit of $\Gamma_{A}$. Since $\operatorname{End}_{A}(U)$ and $\operatorname{End}_{A}(V)$ are division rings by Lemma 2, we conclude from $[2, \S 3]$ that $\operatorname{End}_{A}(U) \cong \operatorname{End}_{A}(V)$. In particular, $q_{A}([U])=q_{A}([V])$. Under our assumption the indecomposable modules in $\Gamma_{A}$ are determined by their composition factors [3]. So we see now that each $\tau$-orbit of $\Gamma_{A}$ contains only finitely many modules. By Lemma 4 the artin algebra $A$ is of finite representation type.

Combining the above theorem with $[7,(4.4)]$, we get the following immediate consequence.

Corollary. Let $A$ be an artin algebra such that $\bmod A$ contains no short $c y$ cle. If there exists a sincere indecomposable module in $\bmod A$, then $A$ is a tilted algebra of finite representation type. In particular, $A$ is of global dimension at most two, and the indecomposable A-modules are determined by their composition factors.

\section{ACKNOWLEDGMENTS}

This work was done during the author's stay in Trondheim. They thank Idun Reiten and Sverre Smalø for their hospitality the referee for a short cut in our original proof of Lemma 3.

\section{REFERENCES}

1. M. Auslander, Applications of morphisms determined by objects, Representation Theory of Algebras, Proc. of the Philadelphia Conference (R. Gordan, ed.), Lecture Notes in Pure and Appl. Math., vol. 37, Dekker, New York, 1976, pp. 245-294.

2. M. Auslander and I. Reiten, Representation theory of artin algebras. III, Comm. Algebra 5 (1975), 239-294.

3. 280-301.

4. D. Happel, U. Preiser, and C. M. Ringel, Vinberg's characterization of Dynkin diagrams using subadditive functions with application to DTr-periodic modules, Lecture Notes in Math., vol. 832, Springer, New York, 1980, pp. 280-294.

5. D. Happel and C. M. Ringel, Directing projective modules, Arch. Math. 60 (1993), 237-249.

6. S. Liu, The degrees of irreducible maps and the shapes of Auslander-Reiten quivers, J. London Math. Soc. (2) 45 (1992), 32-54. 
7. I. Reiten, A. Skowronski, and S. O. Smalø, Short chains and short cycles of modules, Proc. Amer. Math. Soc. 117 (1993), 343-354.

8. C. M. Ringel, Tame algebras and integral quadratic forms, Lecture Notes in Math., vol. 1099, Springer, New York, 1984.

Department of Mathematics and Statistics, University of Trondheim, AVH, 7055 TRONDHEIM, NORWAY

E-mail address, D. Happel: happel@mathematik.tu-chemnitz.de

E-mail address, S. Lin: MATLIUSP@NUSVIT.bitnet 